\title{
Diagnostic Performance of Three Rapid Diagnostic Test Kits for Malaria Parasite Plasmodium falciparum
}

\author{
Seo Hye Park ${ }^{1, \dagger}$, Seung Jegal ${ }^{2, \dagger}$, Seong Kyu Ahn', Haneul Jung ${ }^{1}$, Jinyoung Lee', Byoung-Kuk Na ${ }^{3,4}$, \\ Sung-Jong Hong ${ }^{5}$, Young Yil Bahk ${ }^{6, *}$, Tong-Soo Kim ${ }^{1, *}$ \\ ${ }^{1}$ Department of Tropical Medicine \& Global Resource Bank of Parasitic Protozoa Pathogens, Inha University School of Medicine, Incheon 22212, \\ Korea; '2Department of Infectious Diseases Diagnosis, Incheon Metropolitan City Institute of Public Health and Environment, Incheon 22320, Korea; \\ ${ }^{3}$ Department of Parasitology and Tropical Medicine, and Institute of Health Sciences, Gyeongsang National University School of Medicine, Jinju \\ 52828, Korea; ${ }^{4}$ BK21Plus Team for Anti-aging Biotechnology and Industry, Department of Convergence Medical Science, Gyeongsang National \\ University, Jinju 2828, Korea; ${ }^{5}$ Department of Medical Environmental Biology, Chung-Ang University College of Medicine, Seoul 06974, Korea; \\ ${ }^{6}$ Department of Biotechnology, College of Biomedical and Health Science, Konkuk University, Chungju 27478, Korea
}

\begin{abstract}
Malaria is a potent burden on public healthcare worldwide due to requiring rapid diagnosis and treatment. Nowadays, prompt diagnosis with rapid diagnostic tests (RDTs) has been widely accepted as an effective diagnostic technique in malaria-endemic countries, primarily due to their easy operation, fast output, and straightforward interpretation. The global availability and use of RDTs have gradually grown over recent decades as field-applicable diagnostic tests for the reliable confirmation of malaria infection and proper case management. This study was conducted to evaluate diagnostic performance of 3 commercially available malaria RDT kits : BIOCREDIT ${ }^{\mathrm{TM}}$ Malaria Ag Pf(pLDH), Malaria Ag $P f(\mathrm{pLDH} / \mathrm{pHRPII})$, and Malaria Ag Pf/Pv(pLDH/pLDH) (where pLDH and pHRPII stand for plasmodium lactate dehydrogenase and histidine-rich protein 2, respectively) for the specific detection of Plasmodium falciparum. A total of 1,129 blood samples including 95 blood samples, confirmed as vivax malaria infection by microscopic examinations and a nestedPCR method, were tested for falciparum malaria infection. The overall sensitivity and specificity of Malaria Ag Pf(pLDH/ pHRPII), Malaria Ag Pf/Pv(pLDH/pLDH), and Pf(pLDH) for P. falciparum were $99.0 \%$ and 100\%, 95.8\% and 100\%, and $100 \%$ and $100 \%$, respectively. It is proposed that the 3 RDT kits perform reliable level of diagnostic accuracy of detection for $P$. falciparum parasites.
\end{abstract}

Key words: Plasmodium falciparum, malaria, rapid diagnostic test, diagnostic performance

\section{INTRODUCTION}

Malaria is one of the oldest known and most prevalent parasitic diseases with an estimated 3.2 billion people being at risk of infection [1]. Despite significant control efforts, morbidity and mortality induced by malaria remain high in many developing countries, especially in areas characterized by tropical and subtropical ecosystems [2-5]. In practice, the accurate diagnosis of malaria is the primary tool for effecting rational therapy. Still, access to adequate diagnosis and treatment is insufficient, resulting in a large treatment gap where many cases

\footnotetext{
- Received 27 August 2019, revised 16 March 2020, accepted 21 March 2020.

* Corresponding authors

(bahk12@empal.com, byoung1@kku.ac.kr; tongsookim@inha.ac.kr)

†These authors contributed equally to this work.

(C) 2020, Korean Society for Parasitology and Tropical Medicine

This is an Open Access article distributed under the terms of the Creative Commons Attribution Non-Commercial License (https://creativecommons.org/licenses/by-nc/4.0) which permits unrestricted non-commercial use, distribution, and reproduction in any medium, provided the original work is properly cited.
}

are managed sub-optimally or even go untreated. Thus, it is pivotal to have sensitive and specific malaria diagnostic tools to prevent the injudicious use of anti-malaria drugs and overtreatment. The microscopic examination of Giemsa-, Wright-, or Wright-Giemsa-stained blood smears as a routine reference test has been used as the gold standard for malaria diagnosis in many malaria-endemic areas despite the infrastructural and technical requirements that are not always available in resource-limited settings [6-8]. Thus, many medical resources cannot offer round-the-clock dependable smear-based diagnosis. Inconsistency due to intense inter-observer variability, particularly for samples with low parasitemia or mixed Plasmodium species, has been regarded as a major shortcoming of microscopic examination [8]. These limitations have fostered the development of non-microscopic alternatives for the diagnosis of malaria, especially in field diagnosis [9-12].

Although the World Health Organization (WHO) launched a comparative study of some rapid diagnostic kits (RDTs) on 
selected samples containing P. falciparum and P. vivax in 2008 [13], studies using clinical samples have been highly informative regarding test performance in routine usage. In practice, malaria RDTs from different companies can show wide variation, especially in terms of performance characteristics, and can be affected by many factors that potentially cause falsenegative results [14]. This study was performed to determine functionality of 3 commercially available RDTs Malaria Ag $P f(\mathrm{pLDH})$, Malaria Ag $P f(\mathrm{pLDH} / \mathrm{pHRPI})$, and Malaria Ag Pf/ $P v(\mathrm{pLDH} / \mathrm{pLDH})$ for specific detection of $P$. falciparum.

\section{MATERIALS AND METHODS}

\section{Ethics statements}

The anonymous samples used in this study were donated from the Global Resource Bank of Parasitic Protozoa Pathogens at the Inha University School of Medicine (NRF-2017M3A9B8069530), some of which were exempted from Institutional Review Board (IRB) review and the rest were frozen resources used after the IRB had approved the research (Inha 1712081A).

\section{Samples}

The blood samples used in this study were retrieved from the $-80^{\circ} \mathrm{C}$ stock in Department of Tropical Medicine, Inha University School of Medicine. The samples used in this study were confirmed using $18 \mathrm{~S}$ ribosomal RNA-based genus-specific nested-PCR analyses for $P$. falciparum, P. vivax, P. malariae, and $P$. ovale [15]. The P. falciparum-positive samples were of 191 Ugandan patients [16]. The P. vivax-positive samples were collected from 95 Korean patients by the Global Resource Bank of Parasitic Protozoa Pathogens in the Inha University School of Medicine. The Plasmodium-negative blood samples were of 843 healthy people. Positive samples of $P$. malariae and $P$. ovale were excluded from this study.

\section{Analysis on functionality of RDTs}

All blood samples were assayed with each of the 3 BIOCREDIT $^{\mathrm{TM}}$ Malaria RDTs: Malaria Ag Pf/Pv(pLDH/pLDH) (Lot. No. H016005), Malaria Ag Pf(pLDH) (Lot. No. H019005), and Malaria Ag Pf(pLDH/pHRPII) (Lot. No. H052005) manufactured by RapiGEN Inc. (Gunpo, Korea). These kits were designed to detect $P$. falciparum-specific $\mathrm{pLDH}$ and $P$. vivax-specific pLDH, $P$. falciparum-specific $\mathrm{pLDH}$, and $P$. falciparum-specific pLDH and pHRPII, respectively. Each RDT comprised a membrane strip pre-coated with antibodies specific to each target protein and was tested according to the manufacturer's instructions. In brief, $5 \mu$ of whole blood was loaded into the sample well of the device and 3 drops of assay diluent were added to the buffer well. The test results, interpreted within $35 \mathrm{~min}$, were recorded as negative if only the control line appeared, which indicates that the test had been performed well. Positive or negative results for the respective malaria parasites are based on the color code shown on the strip regardless of test line intensity. Each sample was blinded against the results obtained from the other diagnostic tests. All kits were tested, and the results validated to ensure strict adherence to the manufacturer's statements.

\section{Limit of detection (LOD)}

Samples with known parasite counts per $\mu \mathrm{l}$ of whole blood determined by an expert research microscopist were used to test the LOD of the 3 Malaria RDTs. Three samples of each relevant Plasmodium spp. were chosen: for P. falciparum, 5,440

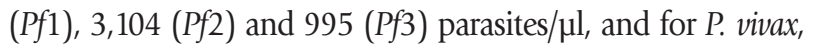

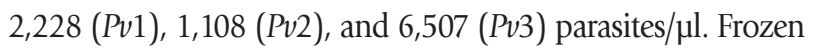
whole blood samples were thawed and then diluted in fresh malaria-negative, non-endemic country whole blood to produce parasitemia with the diluting blood serving as a negative control by serial dilution.

\section{Data analysis}

The 3 sets of RDT results were compared with the microscopic results as the gold standard. The sensitivity, specificity, positive-predictive value (PPV), and negative-predictive value (NPV) of each RDT were calculated. Variable measures were number of true positives (TP), true negatives (TN), false positives (FP), and false negatives (FN). Sensitivity was calculated as $\mathrm{TP} /(\mathrm{TP}+\mathrm{FN})$, specificity as $\mathrm{TN} /(\mathrm{TN}+\mathrm{FP}), \mathrm{PPV}$ as $\mathrm{TP} /(\mathrm{TP}+\mathrm{FP})$, and $\mathrm{NPV}$ as $\mathrm{TN} /(\mathrm{TN}+\mathrm{FN})[17]$.

\section{RESULTS}

Ten out of $191 P$. falciparum-positive samples were negative for the $P f(\mathrm{pLDH})$ antigen in Malaria Ag $P f(\mathrm{pLDH})$ (Table 1), 10/191 positive samples were negative for the $P f(\mathrm{pLDH})$ antigen and 2/191 positive ones were negative for the Pf(pHRPII) antigen in Malaria Ag Pf(pLDH/pHRPII) (Table1), and 8/191 positive samples were negative for the $P f(\mathrm{pLDH})$ antigen in Malaria Ag $P f / P v(\mathrm{pLDH} / \mathrm{pLDH})$ (Table 2). Sensitivity and specificity of Malaria $\mathrm{Ag} P f(\mathrm{pLDH})$ were $94.8 \%$ and $100 \%$, respec- 
Table 1. Comparison of $P$. falciparum detection by Malaria Ag Pf(pLDH/pHRPII) and microscopic examination $(n=1,034)$

\begin{tabular}{|c|c|c|c|c|c|}
\hline \multirow{2}{*}{$\begin{array}{l}\text { Microscopic examination } \\
\text { Result }\end{array}$} & \multirow{2}{*}{ No. sample } & \multicolumn{2}{|c|}{$P f(p L D H)$} & \multicolumn{2}{|c|}{$P f($ pHRPII) } \\
\hline & & No. positive & No. negative & No. positive & No. negative \\
\hline Positive & 191 & 181 & 10 & 189 & 2 \\
\hline Negative & 843 & 0 & 843 & 0 & 843 \\
\hline Total & 1,034 & 181 & 853 & 189 & 845 \\
\hline
\end{tabular}

Table 2. Comparison of $P$. falciparum and $P$. vivax detection by Malaria Ag $P f / P v(p L D H / p L D H)$ and microscopic examination $(n=1,129)$

\begin{tabular}{|c|c|c|c|c|c|}
\hline \multirow{2}{*}{$\begin{array}{l}\text { Microscopic examination* } \\
\text { Result }\end{array}$} & \multirow{2}{*}{ No. sample } & \multicolumn{2}{|c|}{$P f(p L D H)$} & \multicolumn{2}{|c|}{$P v(\mathrm{pLDH})$} \\
\hline & & No. positive & No. negative & No. positive & No. negative \\
\hline P. falciparum & 191 & 183 & 8 & 0 & 0 \\
\hline P. vivax & 95 & 0 & 0 & 95 & 0 \\
\hline Negative & 843 & 0 & 843 & 0 & 843 \\
\hline Total & 1,129 & 183 & 851 & 95 & 843 \\
\hline
\end{tabular}

*These samples were tested and confirmed using nested-PCR.

Table 3. Performance of Malaria Ag Pf(pLDH/pHRPII) compared to microscopic examination $(n=1,034)$

\begin{tabular}{lccccc}
\hline RDT & Sensitivity (\%) (95\% Cl*) & Specificity (\%) (95\% Cl) & PPV (\%) (95\% Cl) & NPV (\%) (95\% Cl) & Accuracy (\%) (95\% Cl) \\
\hline Pf(pLDH) & $94.8(90.6-97.5)$ & $100(97.6-100)$ & 100 & $93.8(97.98-99.4)$ & $99.2(98.2-99.5)$ \\
Pf(pHRPII) & $99.0(96.3-99.9)$ & $100(99.6-100)$ & 100 & $99.8(99.1-99.9)$ & $99.8(99.3-100)$ \\
Pf(pLDH/pHRPII) & $99.0(96.3-99.9)$ & $100(99.6-100)$ & 100 & $99.8(99.1-99.9)$ & $99.8(99.3-100)$ \\
\hline
\end{tabular}

${ }^{*}$ Confidence interval.

Table 4. Performance comparisonof Malaria Ag Pf/Pv(pLDH/pLDH) microscopic examination $(n=1,129)$

\begin{tabular}{lccccc}
\hline & Sensitivity (\%) (95\% Cl*) & Specificity (\%) (95\% Cl) & PPV(\%) (95\% Cl) & NPV(\%) (95\% Cl) & Accuracy (\%) (95\% Cl) \\
\hline$P f(p L D H)$ & $95.8(91.9-98.2)$ & $100(97.6-100)$ & 100 & $99.1(98.2-99.5)$ & $99.2(98.5-99.7)$ \\
$P V(p L D H)$ & $100(96.2-100)$ & $100(99.6-100)$ & 100 & $100(99.6-100)$ & $100(99.6-100)$ \\
\hline
\end{tabular}

tively (Table 3). Malaria Ag Pf(pLDH/pHRPII) showed sensitivity $94.8 \%$ and specificity $100 \%$ for $P f(\mathrm{pLDH})$, and $99.0 \%$ and $100 \%$ for Pf(pHRPII). Overall sensitivity and specificity were 99.0\% and 100\%, respectively (Table 3). Those of Malaria Ag $P f / P v(\mathrm{pLDH} / \mathrm{pLDH})$ were $95.8 \%$ and $100 \%$ for $P f(\mathrm{pLDH})$, and $100 \%$ and $100 \%$ for $P v(\mathrm{pLDH})$, respectively (Table 4 ).

PPV and NPV of Malaria Ag Pf(pLDH) were 100\% and 93.8\% (Table 3), while those of Malaria Ag Pf(pLDH/pHRPII) were $100 \%$ and $98.8 \%$ for $P f(\mathrm{pLDH})$, and $100 \%$ and $99.8 \%$ for Pf(pHRPII), respectively (Table 3). Those of Malaria Ag Pf/ $P v(\mathrm{pLDH} / \mathrm{pLDH})$ were $100 \%$ and $99.1 \%$ for $P f(\mathrm{pLDH})$ and $100 \%$ and $100 \%$ for $P v(\mathrm{pLDH})$, respectively (Table 4 ).

LODs for P. falciparum with Malaria Ag Pf(pLDH) and Malaria Ag Pf(pLDH/pHRPII) RDTs were 8.4 and 10.1 parasites/ $\mu \mathrm{l}$ on average, respectively. LODs for $P$. falciparum and $P$. vivax with Malaria Ag Pf/Pv(pLDH/pLDH) RDT were 7.6 and 38.2 parasites/ $\mu$ l on average, respectively (Table 5).
Table 5. Detection limit of 3 Malaria Ag kits

\begin{tabular}{|c|c|c|c|}
\hline RDT kit & Sample & Test antigen & LOD (parasites/ $/$ l) \\
\hline Malaria Ag Pf(pLDH) & $\begin{array}{l}P f 1 \\
P f 2 \\
P f 3\end{array}$ & $\begin{array}{l}\text { PfLDH } \\
\text { PfLDH } \\
\text { PfLDH }\end{array}$ & $\begin{array}{r}13.0 \\
7.5 \\
4.7\end{array}$ \\
\hline Malaria Ag Pf(pLDH/pHRPII) & $\begin{array}{l}P f 1 \\
P f 2 \\
P f 3\end{array}$ & $\begin{array}{l}\text { PfpLDH } \\
\text { PfHRPII } \\
\text { PfpLDH } \\
\text { PfHRPII } \\
\text { PfpLDH } \\
\text { PfHRPII }\end{array}$ & $\begin{array}{r}13.0 \\
13.0 \\
15.0 \\
15.0 \\
9.5 \\
2.4\end{array}$ \\
\hline Malaria Ag Pf/Pv(pLDH/pLDH) & 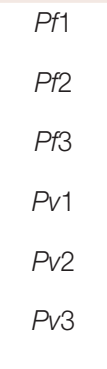 & $\begin{array}{l}P f \\
P V \\
P f \\
P V \\
P f \\
P V \\
P f \\
P V \\
P f \\
P V \\
P f \\
P V\end{array}$ & $\begin{array}{c}3.2 \\
\text { Negative } \\
15.0 \\
\text { Negative } \\
4.7 \\
\text { Negative } \\
\text { Negative } \\
42.2 \\
\text { Negative } \\
40.6 \\
\text { Negative } \\
31.7\end{array}$ \\
\hline
\end{tabular}

*Three samples were chosen each from Plasmodium sp.: for $P$. falciparum, 5,440 (Pf1), 3,104 (Pf2) and 995 (Pf3) parasites/ $\mu$, and for $P$. vivax, 2,228 (Pv1), 1,108 (Pv2), and 6,507 (Pv3) parasites/ul. 


\section{DISCUSSION}

In the present study, we examined the ability of the Malaria Ag RDTs to diagnose falciparum malaria. To determine the appropriate test for field use for $P$. falciparum, study population consisted of 2 groups of patients from Uganda for falciparum malaria and from Korea for vivax malaria. According to WHO recommendation [4], RDTs should show greater than 95\% sensitivity to be useful and efficient diagnostic tools.

The WHO estimates that 219 million cases occurred during 2017 with 435,000 deaths [18]. Most malaria cases in 2017 were in Africa (approximately 200 million or 92\%), followed by South-East Asia (5\%) and the Eastern Mediterranean region (2\%). Prompt diagnosis and treatment is the most effective means to prevent a mild case of malaria from advancing into severe disease and even death. Moreover, the clinical characteristics of malaria are nonspecific and overlap primarily with those of other febrile diseases. Thus, the WHO recommends that all patients should have a parasite-specific laboratory test performed to confirm the clinical symptoms. P. falciparum is the most prevalent malaria parasite in Africa, accounting for $99.7 \%$ of estimated malaria cases in 2017, as well as in SouthEast Asia (62.8\%), and the Eastern Mediterranean (69\%) and the Western Pacific regions (71.9\%) [18].

In the USA, a malaria test is the only one cleared by the Food and Drug Administration for the in-vitro diagnosis of malaria. That malaria test has sensitivities of $100 \%$ and $81.6 \%$ for the detection of $P$. falciparum and $P$. vivax, respectively, in venous blood samples [19]. According to the WHO recommendations for RDT performance, only 2 RDTs with a reported sensitivity greater than 95\% have been approved: $96.8 \%$ and $95.2 \%$ [20]. However, present study showed that sensitivity of the 3 Malaria Ag kits was $99.0 \%$ overall when using Pf(pLDH/pHRPII) RDT kit. This kit had high sensitivity and was in strong agreement with the microscopic examinations and the 18s ribosomal RNA-based nested-PCR results. HRPII-based RDTs commonly provide P. falciparum sensitivity higher than $90.0 \%$ in clinical cases $[21,22]$. However, the test results with pLDH assays have been shown to vary among studies $[4,23]$. In the present study, antigen $P f(\mathrm{pLDH})$ in the Malaria $\mathrm{Ag} P f(\mathrm{pLDH})$ and $P f(\mathrm{pLDH} /$ pHRPII) RDTs showed $94.8 \%$ sensitivity. Interestingly, sensitivity of Malaria $\mathrm{Ag} P f(\mathrm{pLDH} / \mathrm{pHRPII})$ was $99.0 \%$ and that of Malaria $\mathrm{Ag} P f / P v(\mathrm{pLDH})$ was $95.8 \%$. It is understandable that RDTs can be influenced by several factors such as antigenic variability of the target protein, antigen persistence and parasite density in blood samples [24,25].

Most studies on the diagnostic serology of Plasmodium spp. include immunoassays using limited antigen, such as HRPII. Nowadays, these almost always contain the highly conserved immunodominant epitope of HRPII at a minimum. Despite the earlier detection of Plasmodium spp. in patients' blood samples, this first-generation RDT kit using HRPII has shown decreased sensitivity in detecting malaria infection. In general, PfHRPII-based RDTs are more sensitive and heat-stable than RDTs that detect other malaria antigens, such as pan (all species) or P. falciparum-specific pLDH or aldolase [26,27]. However, antibodies against PfHRPII can cross-react with proteins expressed by another member of the hrp gene family such as pfhrp3 due to a strong similarity in the amino acid sequences. PfHRPII RDTs also have limitations due to the genetic variability and polymorphism of the pfhrp2 gene, which can affect its detection by RDTs [28-30]. The prevalence of $p f h r p 2$ gene deletion varies from location to location [31] and strains with partial or total pfhrp2 deletion have been reported in South America, Africa, and India [32]. Moreover, a recent study in India reported a $2.4 \%$ prevalence of $p f h r p 2$ gene deletion [33]. These genetic variations in the region of the $p f h r p 2$ gene have caused a high rate of false-negative results when using RDTs, and the companies that make them are under pressure to develop new specific antigenic proteins as useful and essential target(s) for $P$. falciparum detection. Furthermore, RDTs used to detect malaria in pregnant women can show low sensitivity, possibly due to the sequestration of antigens in the placental circulation [34]. Therefore, it is critical to develop and improve alternative biomarkers of $P$. falciparum for the next generation RDTs for malaria parasite detection [35]. Thus, in the present study, we demonstrated that the monoclonal antibodies against $\mathrm{pLDH}$ in 3 commercially available 2nd generation Malaria Ag RDTs are better candidates for diagnosing falciparum malaria infection than the 1st generation HRPII-based RDT kits.

Previously we evaluated the diagnostic performances of 2 commercially available malaria RDT kits, Malaria Ag Pf/ $P v(\mathrm{pLDH} / \mathrm{pLDH})$ and competitor's Ag $P f / P v(\mathrm{pHRPII} / \mathrm{pLDH})$ for detectability of Plasmodium species in blood samples collected from Ugandan patients with malaria. The detection sensitivity of Malaria Ag $P f / P v(\mathrm{pLDH} / \mathrm{pLDH})$ and competitor for $P$. falciparum was $87.8 \%$ and $89.6 \%$, respectively, and the specificities of the 2 RDTs were $100 \%$ for $P$. falciparum and mixed $P$. falciparum/P. vivax samples [8]. A high panel detection scores were shown with other kits, even at low parasitemia, in Round 
4 of the WHO/FIND study [26]. The sensitivity and specificity of the RDTs assayed in this study were higher than estimates of those previously developed commercial RDTs. Although their diagnostic performances in a field setting have yet not been established, these Malaria Ag kits provided good diagnostic performances with $P$. falciparum/P. vivax -positive blood samples at a laboratory setting. Considering their performance results, we recommend these RDT kits as an appropriate option for screening for $P$. falciparum at health facilities with limited human resources and infrastructure.

In conclusion, we evaluated the clinical performance of $3 \mathrm{Ma}-$ laria Ag kits for P. falciparum using whole blood samples compared to microscopic examination as the gold standard and molecular nested-PCR tests. The accuracies of the RDTs were similar to or better than those of the RDTs currently recommended by WHO [20]. Therefore, Malaria Ag kits were shown to be reliable diagnostic kits to detect falciparum malaria infections and can contribute to malaria control efforts as a possible replacement for microscopic examination in front-line diagnosis. For further studies, more extensive tests with mixed infections of $P$. falciparum and $P$. vivax and with low parasitemia values from various global populations are needed to fully evaluate the diagnostic performance of the 3 Malaria Ag kits.

\section{ACKNOWLEDGMENTS}

This research was supported by grants from the NRF of Korea (NRF-2018R1D1A1B07047721, YYB), by the Inha University Research Fund (TSK) and through the Global Resource Bank of Parasitic Protozoa Pathogens of Inha University School of Medicine (NRF-2017M3A9B8069530, TSK).

\section{CONFLICT OF INTEREST}

The authors declare that they have no conflicts of interest.

\section{REFERENCES}

1. Centers for Disease Control and Prevention. The History of Malaria, An Ancient Disease [Internet]; Washington DC, USA: Available from: https://www.cdc.gov/malaria/about/history/.

2. Singh B, Daneshvar C. Human infections and detection of Plasmodium knowlesi. Clin Microbiol Rev 2013; 26: 165-184.

3. Rubio JM, Buhigas M, Subirats M, Baquero M, Puente S, Benito A. Limited level of accuracy provided by available rapid diagnosis tests for malaria enhances the need for PCR-based reference lab- oratories. J Clin Microbiol 2001; 39: 2736-2737.

4. World Health Organization. Malaria Diagnosis New Perspectives: Report of the Joint WHO/USAID Informal Consultation. Geneva, Switzerland. World Health Organization. 1999.

5. Casalino E, Le Bras J, Chaussin F., Fichelle A, Bouvet E. Predictive factors of malaria in travelers to areas where malaria is endemic. Arch Intern Med 2002; 162: 1625-1630.

6. Garcia LS, Isenberg HD. Clinical Microbiology Procedures Handbook. 2nd ed. Washington, DC. ASM Press. 2007.

7. Mathison BA, Pritt BS. Update on malaria diagnostics and test utilization. J Clin Microbiol 2017; 55: 2009-2017.

8. Bahk YY, Park SH, Lee W, Jin K, Ahn SK, Na BK, Kim TS. Comparative assessment of diagnostic performances of two commercial rapid diagnostic test kits for detection of Plasmodium spp. in Ugandan patients with malaria. Korean J Parasitol 2018; 56: 447-452.

9. World Health Organization. Information Note on Recommended Selection Criteria for Procurement of Malaria Rapid Diagnostic Tests (RDTs). Geneva, Switzerland. World Health Organization. 2016.

10. Moody AH. Rapid diagnostic tests for malaria parasites. Clin Microbiol Rev 2001; 15: 66-78.

11. Incardona S, Serra-Casas E, Champouillon N, Nsanzabana C, Cunningham J, González IJ. Global survey of malaria rapid diagnostic test (RDT) sales, procurement and lot verification practices: assessing the use of the WHO-FIND malaria RDT evaluation programme (2011-2014). Malar J 2017; 16: 196.

12. Chilton D, Malik AN, Armstrong M, Kettelhut M, Parker-Williams J, Chiodini PL. Use of rapid diagnostic tests for diagnosis of malaria in the UK. J Clin Pathol 2006; 59: 862-866.

13. World Health Organization. Malaria Rapid Diagnostic Test Performance. Summary Results of WHO Product Testing of Malaria RDTs: Round 1-4 (2008-2012) [Internet]. Available: https://www. who.int/malaria/publications/rdtmalaria_summary.pdf.

14. World Health Organization. False-Negative RDT Results and Implications of New Reports of P. falciparum histidine-rich protein 2/3 Gene Deletions [Internet]. Available: https://apps.who.int/iris/bitstream/handle/10665/258972/WHO-HTM-GMP-2017.18-eng. pdf.

15. Singh B, Bobogare A, Cox-Singh J, Snounou G, Abdullah MS, Rahman HA. A genus- and species-specific nested polymerase chain reaction malaria detection assay for epidemiologic studies. Am J Trop Med Hyg 1999; 60: 687-692.

16. Bahk YY, Cho PY, Ahn SK, Lee WJ, Kim TS. An evaluation of active case detection in malaria control program in Kiyuni Parish of Kyanwanzi district, Uganda. Korean J Parasitol 2018; 56: 625632.

17. Tjitra E, Suprianto S, Dyer M, Currie BJ, Anstey NM. Field evaluation of the ICT malaria $P f / P v$ immunochromatographic test for detection of Plasmodium falciparum and Plasmodium vivax in patients with a presumptive clinical diagnosis of malaria in Eastern Indonesia. J Clin Microbiol 1999; 37: 2412-2417.

18. World Health Organization. World Malaria Report 2018. Gene- 
va, Switzerland. World Health Organization.

19. BinaxNOW. Malaria Test Kit [Internet]. Lake Bluff, Illinois, USA: Available from: http://www.alere.com/en/home/product-details/ binaxnow-malaria.html.

20. World Health Organization. Malaria Rapid Diagnosis: Making it Work. Geneva, Switzerland. World Health Organization. 2003.

21. Killian AH, Kanagamble G, byamukama W, Langi P, Weis P, von Sonnenburg F. Application of the ParaSight-F dipstick test for malaria diagnosis in a district control program. Acta Trop 1999; 72: 281-293.

22. Guthmann JP, Ruiz A, Priotto G, Kiguli J, Bonte L, Legros D. Validity, reliability and ease of use in the field of five rapid tests for the diagnosis of Plasmodium falciparum malaria in Uganda. Trans R Soc Trop Med Hyg 2002; 96: 254-257.

23. Singh N, Valecha N, Nagpal N, Nagpal AC, Mishra SS, Varma HS, Subbarao SK. The hospital- and field-based performance of the OptiMAL test, for malaria diagnosis and treatment monitoring in central India. Ann Trop Med Parasitol 2003; 97: 5-13.

24. Lee N, Gatton ML, Pelecanos A, Bubb M, Gonzales I, Bell D, Cheng Q, McCarthy JS. Identification of optimal epitopes for Plasmodium falciparum rapid diagnostic tests that target histidinerich proteins 2 and 3. J Clin Micriobiol 2012; 50: 1397-1405.

25. Houzé S, Boly MD, Le Bras J, Deloron P, Faucher JE. PfHRP2 and $P f L D H$ antigen detection for monitoring the efficacy of artemisinin-based combination therapy (ACT) in the treatment of uncomplicated falciparum malaria. Malaria J 2009; 8: 211.

26. World Health Organization. Malaria Rapid Diagnostic Test Performance. Results of WHO Product Testing of Malaria RDTs: Round 4. Geneva, Switzerland. World Health Organization. 2012.

27. World Health Organization. Malaria Rapid Diagnostic Test Performance. Summary Results of WHO Product Testing of Malaria RDTs: Round 1-5 (2008-2013). Geneva, Switzerland. World
Health Organization. 2014

28. Mariette N, Bamadas C, Bouchier C, Tichit M, Ménard D. Country-wide assessment of the genetic polymorphism in Plasmodium falciparum and Plasmodium vivax antigens detected with rapid diagnostic tests for malaria. Malar J 2008; 7: 219.

29. Nima MK, Hougard T, Hossain ME, Kibria MG, Mohon AN, Johora FT, Rahman R, Haque R, Alam MS. Case report: a case of Plasmodium falciparum hrp2 and $h r p 3$ gene mutation in Bangladesh. Am J Trop Med. Hyg 2017; 97: 1155-1158.

30. Berhane A, Anderson K, Mihreteab S, Gresty K, Rogier E, Mohamed S, Hagos F, Embaye G, Chinorumba A, Zehaie A, Dowd S, Waters NC, Gatton ML, Udhayakumar V, Cheng Q, Cunningham J. Major threat to malaria control programs by Plasmodium falciparum lacking histidine-rich protein 2, Eritrea. Emerg Infect Dis 2018; 24: 462-470.

31. Cheng Q, Gatton ML, Barnwell J, Chiodini P, McCarthy J, Bell D, Cunningham J. Plasmodium falciparum parasites lacking histidine-rich protein 2 and 3: a review and recommendations for accurate reporting. Malar J 2014; 13: 283.

32. Deme AB, Park DJ, Bei AK, Sarr O, Badiane AS, Gueye Pel H, Ahouidi A, Ndir O, Mboup S, Wirth DF, Ndiaye D, Volkman SK. Analysis of $p f h r p 2$ genetic diversity in Senegal and implications for use of rapid diagnostic tests. Malar J 2014; 13: 34.

33. Bharti PK, Chandel HS, Ahmad A, Krishna S, Udhayakumar V, Singh N. Prevalence of $p f h r p 2$ and/or pfhrp3 gene deletion in Plasmodium falciparum population in eight highly endemic states in India. PLoS One 2016; 11: e0157949.

34. Fried M, Muehelnbachs A, Duffy PE. Diagnosing malaria in pregnancy: an update. Expert Rev Anti Infect Ther 2012; 10: 1177-1187.

35. Piper RC, Buchanan I, Choi YH, Makler MT. Opportunities for improving pLDH-based malaria diagnostic tests. Malar J 2011; 10: 213. 